\title{
Evaluación del rendimiento del coprocultivo en pacientes hospitalizados
}

\author{
ANTONIETA BLU F., EUGENIA LEÓN C., JUAN C. ROMÁN G., \\ LEONARDO CHANQUEO C. y PATRICIA GARCÍA C.
}

\section{Utility of stool culture in inpatient}

Several studies have concluded that it is inappropriate to perform stool cultures (SC) to inpatients that have stayed hospitalized three days or more because nosocomial diarrhea is not due to enteric pathogenic bacteria that are searched through this exam. The aim of this paper was to analyze the SC yield performed to patients hospitalized in the UC Health Net since January to December 2002 and the rate of positive results obtained depending on the length of hospitalization in order to define if international guidelines are useful to the national reality. During twelve months 3474 SC were evaluated, $458(13.2 \%)$ belonged to inpatients. Of them $16(3.5 \%)$ were positive, 13 were obtained on the first day, 2 at the second day and 1 at the fifth day of hospitalization. Only 1/190 (0.5) SC obtained after two days of hospitalization yielded a positive result. These results reinforce the international recommendation of not performing SC to inpatients that have stayed more than three days at the hospital; besides, considering previous figures, we suggest to make a cutoff at day two of hospitalization, after two days there is no a significant clinical impact. This strategy means to reduce $41 \%$ of these analyses and to save the corresponding money.

Key words: Stool culture; Nosocomial diarrhea; Enteric pathogenic bacteria.

Palabras clave: Coprocultivo, diarrea nosocomial, enteropatógenos.

\section{Introducción}

El síndrome diarreico agudo es una causa muy frecuente de consulta y hospitalización, sobre todo en niños. Generalmente la causa de estos cuadros se ha asociado a malas condiciones de higiene o a una inadecuada preparación y conservación de los alimentos. Sin embargo, en pacientes hospitalizados la diarrea generalmente es producida por otras causas. Se ha visto que en lactantes, entre 20 y $50 \%$ de las diarreas nosocomiales pueden ser causadas por rotavirus o astrovirus $^{4}$. Escherichia coli enteropatógena puede ser causa de brotes intrahospitalarios por su alta transmisibilidad; sin embargo, se recomienda su búsqueda sólo en brotes epidémicos. ${ }^{5}$ Clostridium difficile, también es un agente cau- sal de gran importancia en diarrea nosocomial. La prevalencia de este último en muestras de pacientes hospitalizados con diarrea puede llegar hasta $10 \%$.

En cuanto a las bacterias enteropatógenas que habitualmente se buscan en el coprocultivo (CP), la tasa de positividad de las muestras obtenidas de pacientes que llevan menos de tres días hospitalizados puede ser de hasta $12 \%$; sin embargo, esta cifra disminuye a $1,4 \%$ en pacientes que llevan tres días o más hospitalizados ${ }^{7}$, siendo este último grupo de pacientes el que contribuye hasta en $50 \%$ del total de $\mathrm{CP}$ realizados ${ }^{1}$.

Dado que el CP es un examen laborioso y de alto costo, es necesario racionalizar su indicación, seleccionando los pacientes que clínicamente o por situación epidemiológica lo ameriten. Habi-

Facultad de Medicina, Pontificia Universidad Católica de Chile; Santiago, Chile.

Estudiante de Medicina (ABF).

Laboratorio de Microbiología. UDA Laboratorios Clínicos (ELC, JCRG, PGC).

Residente Infectología. Departamento de Medicina Interna (LChC). 
tualmente se sugiere realizar $\mathrm{CP}$ en pacientes ambulatorios con diarrea severa, que no ceda al tratamiento sintomático, cuando hay sangre en las deposiciones, en diarrea prolongada en pacientes inmunocomprometidos, si existen antecedentes de viajes, en pacientes con leucocitosis con neutrofilia y en pacientes desnutridos ${ }^{8}$.

Los mayores predictores de positividad en un CP son: diarrea por más de 24 horas, fiebre, dolor abdominal y sangre en las deposiciones?. En el caso de pacientes hospitalizados es necesario tomar $\mathrm{CP}$ en los estudios de brotes de gastroenteritis ${ }^{5}$ por lo que no se justifica realizar $\mathrm{CP}$ en los pacientes cuyo síndrome diarreico se ha iniciado al tercer día de hospitalización o de allí en adelante $^{3}$, ya que en estos casos, los cuadros diarreicos probablemente no sean causados por los patógenos habitualmente buscados en el CP.

Solamente el hecho de instaurar guías para el uso racional del $\mathrm{CP}$ ha permitido disminuir el número de $\mathrm{CP}$ solicitados de pacientes hospitalizados hasta en $70 \%{ }^{10}$.

En consideración a lo anteriormente expuesto, el objetivo de este trabajo fue conocer el porcentaje de CP provenientes de pacientes hospitalizados y analizar el rendimiento del examen en este grupo de pacientes, según los días de hospitalización.

\section{Material y Métodos}

Se revisaron retrospectivamente todos los $\mathrm{CP}$ recibidos en el Laboratorio de Microbiología de la Red de Salud UC desde enero a diciembre del 2002. Se separaron según su procedencia en ambulatorios y hospitalizados y en los pacientes hospitalizados se registraron los días de hospitalización. Se revisaron las fichas clínicas de aquellos pacientes con CP positivos, para determinar la causa de ingreso al hospital y el tratamiento que recibieron.

Las muestras se transportaron en medio Cary Blair, (Eurotubo®, I.A.S.A, España) y se sembraron en agar Hektoen (BiomerieuxOे, Ha, MO), Mac Conkey (Biomerieux $\left.{ }^{\circledR}, \mathrm{Ha}, \mathrm{MO}\right)$ y caldo selenito con resiembra en Hektoen después de incubar 16 horas a $35^{\circ} \mathrm{C}^{11}$, para los $\mathrm{CP}$ de todas las edades. En pacientes con menos de 10 años, se agregó siembra en agar Mac Conkey/sorbitol (Laboratorios LINSANOे).

Se buscaron e identificaron por métodos convencionales $^{12}$ Salmonella sp, Shigella sp y Yersinia sp agregándose la búsqueda de Campylobacter por tinción de Hucker según lo descrito por Valenzuela y cols ${ }^{13}$ en los $\mathrm{CP}$ provenientes de todos los pacientes. En niños bajo 10 años de edad se agregó la búsqueda de $E$. coli enterohemorrágica serotipos O26, O111, O157 y O55 utilizando antisueros PROBACÒ (Productos bacteriológicos LTDA, Brasil). Se aglutinó con mezcla I y monovalente O157. En caso que fuese positiva la mezcla I, se utilizó el monovalente O111 y O26. Sólo de ser negativo se continuó con el antisuero O55. Toda aglutinación positiva en lámina se confirmó en tubo, según las recomendaciones de la Sección Bacteriología del Instituto de salud Pública de Chile.

\section{Resultados}

Se recibieron durante el año 2002, $3474 \mathrm{CP}$, de los cuales $3016(86,8 \%)$ provenían de pacientes ambulatorios y $458(13,2 \%)$ de pacientes hospitalizados. De éstos, 119 correspondieron a pacientes con menos de 10 años, con un promedio de edad de 3,6 años; 339 correspondieron a pacientes mayores de 10 años con un promedio de 56,5 años.

La distribución porcentual según los días de hospitalización, de los $458 \mathrm{CP}$ obtenidos de pacientes hospitalizados, y el porcentaje de positividad según los días de hospitalización, se muestran en la Tabla 1.

El rendimiento de los $\mathrm{CP}$ en pacientes hospitalizados fue 3,5\% (16/458), siendo el 94\% de ellos $(15 / 16)$ obtenidos durante el primer y segundo día de hospitalización. Se observa que sólo un CP de $190(0,5 \%)$ resultó positivo en pacientes con más de dos días de hospitalización.

Los agentes etiológicos aislados de los CP positivos de pacientes hospitalizados, según el día de evolución, se muestran en la Tabla 2. En el único paciente que presentó CP positivo después del segundo día, se aisló Salmonella Typhimurium.

El análisis clínico de los pacientes hospitalizados con CP positivos muestra que en $13 \mathrm{el}$ motivo de ingreso fue diarrea o síndrome disentérico. De éstos, en 10 casos se inició tratamiento antimicrobiano empírico antes de recibir el resultado del CP y en los otros 3 no se indicó antibioterapia, siendo manejados con hidratación endovenosa.

Los 3 pacientes restantes, que no ingresaron por diarrea, se describen a continuación. El primero era un paciente de 62 años que ingresó por hematoquezia y pólipos en el colon, en cuyo CP se aisló Pseudomonas aeruginosa. No recibió tratamiento antimicrobiano y su recuperación fue satisfactoria. 
Tabla 1. Coprocultivos obtenidos en pacientes hospitalizados en la Red de Salud de la Pontificia Universidad Católica de Chile, desde enero a diciembre 2002, según días de hospitalización

\begin{tabular}{lcccc}
\hline $\begin{array}{c}\text { Días de } \\
\text { hospitalización }\end{array}$ & CP obtenidos & $\begin{array}{c}\text { \% de CP obtenidos } \\
\text { por día }\end{array}$ & CP positivos & \% de CP positivos \\
\hline 1er día & 163 & $35,6 \%$ & 13 & $7,9 \%$ \\
2do día & 105 & $22,9 \%$ & 2 & $1,9 \%$ \\
3er día & 36 & $7,9 \%$ & 0 & $0 \%$ \\
$4^{\circ}$ día & 23 & $5 \%$ & 0 & $0 \%$ \\
Más de 4 días & 131 & $28,6 \%$ & 1 & $0,76 \%$ \\
Total & 458 & $100 \%$ & 16 & $3,5 \%$ \\
\hline
\end{tabular}

Tabla 2. Total de bacterias enteropatógenas aisladas de coprocultivos de pacientes hospitalizados en la Red de Salud de la Pontificia Universidad Católica de Chile, según día de hospitalización

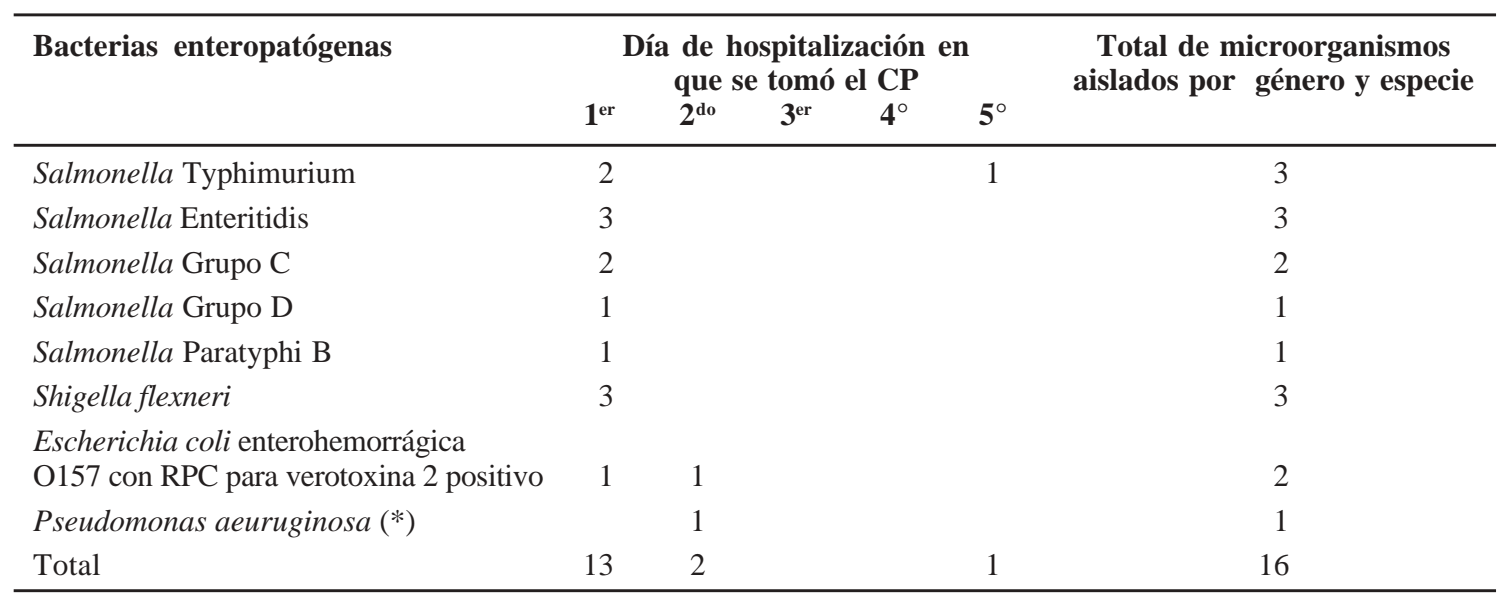

(*) No existe una relación causal clara entre este agente y síndrome diarreico. Sin embargo, la literatura describe que no se puede descartar como epifenómeno en pacientes gravemente enfermos con infecciones extra-intestinales por Pseudomonas ${ }^{15}$.

El segundo caso se trataba de una niña de 6 años que ingresó por anemia hemolítica acompañada de vómitos, sin diarrea clínica. Se sospechó de un síndrome hemolítico urémico por lo que se tomó un $\mathrm{CP}$ del cual se aisló E. coli enterohemorrágica $\mathrm{O} 157$ con RPC positiva para verotoxina 2. No recibió tratamiento antimicrobiano.

El tercer caso fue un paciente de 52 años que ingresara por insuficiencia renal aguda, en encefalopatía urémica, secundaria a foco séptico renal por E. coli. Durante la evolución presentó una pancreatitis aguda y al tercer día de hospitalización comenzó con diarrea y síndrome febril. Se tomó CP al quinto día aislándose Salmonella Typhimurium. La detección de toxina de Clostridium difficile fue negativa. El paciente fue tratado con ciprofloxacina y tuvo una evolución favorable (en este caso Salmonella se aisló a partir del caldo selenito).

\section{Discusión}

Nuestros resultados muestran que sólo $13 \%$ de los CP realizados en un año correspondían a pacientes hospitalizados, a diferencia de lo reportado en E.U.A., en un estudio realizado por el Colegio Americano de Patólogos, en que esta proporción correspondía a la mitad de los $\mathrm{CP}^{3}$.

Es importante destacar que de este $13 \%$ (458 $\mathrm{CP})$, la mayoría $(58,5 \%)$ fueron obtenidos durante los dos primeros días de hospitalización, lo que permitiría suponer que estos pacientes presentaban síndrome diarreico al momento del ingreso al hospital. Además, del total de $\mathrm{CP}$ positivos obtenidos de pacientes hospitalizados, $94 \%$ $(15 / 16)$ fueron obtenidos durante los dos primeros días de hospitalización. Estos datos reflejan que se trataría de pacientes cuya diarrea comenzó en la fase ambulatoria y que posteriormente 
fueron hospitalizados para optimizar el tratamiento o por presentar signos de gravedad, pero que en estricto rigor, no se trataría de diarreas nosocomiales.

En cuanto al rendimiento de los $\mathrm{CP}$ en pacientes hospitalizados por más de 2 días, pudimos observar que sólo uno de éstos fue positivo (1/ 190), aislándose Salmonella Typhimurium, en un paciente con diarrea clínicamente significativa. Si es que se hubiera instaurado un corte a los dos días de hospitalización como máximo para poder tomar el $\mathrm{CP}$, en este caso no se habría confirmado microbiológicamente el agente etiológico; sin embargo, no se habría modificado mayormente la evolución del paciente; ya que como presentaba diarrea, de todas formas se estaba manejando con precauciones de contacto y además, un tratamiento empírico sin conocimiento de la susceptibilidad in vitro de esta cepa, no habría influido negativamente en la recuperación del paciente. Por otro lado, este agente aislado es zoonótico e incluso no está claro que el tratamiento antibacteriano sea de utilidad en las gastroenteritis no complicadas por este agente. Es importante destacar que la muestra fue tomada tardíamente (al $5^{\circ}$ día), ya que el paciente comenzó con diarrea al tercer día, lo que indica que probablemente este diagnóstico se podría haber hecho en forma más precoz. Por último, este agente se aisló sólo a partir de caldo selenito, el que aumenta la sensibilidad para la pesquisa de Salmonella a partir de un bajo número de estos microorganismos.

Creemos que más importante que implementar criterios de rechazo, es difundir en el ámbito médico la correcta selección de los pacientes que, estando hospitalizados, requieran $\mathrm{CP}^{14}$, como son los pacientes inmunocomprometidos, los con edad superior a 65 años y comorbilidad, pacientes infectados por VIH y en los casos de brotes epidémicos intrahospitalarios ${ }^{7}$.

Respecto a los agentes etiológicos encontrados, destaca el aislamiento de $P$. aeruginosa en un paciente; si bien se consideró como agente patógeno, no existe una relación causal clara entre este agente y diarrea nosocomial. La literatura describe que no se puede descartar como epifenómeno en pacientes gravemente enfermos con infecciones extra-intestinales causadas por Pseudomonas ${ }^{15}$.

En los pacientes que inician un cuadro diarreico estando hospitalizados, la búsqueda debe orientarse a $C$. difficile, que ha mostrado una alta incidencia en diarreas nosocomiales en pacientes que están recibiendo antimicrobianos, pacientes sometidos a cirugía gastrointestinal o que han estado hospitalizados por largo tiempo ${ }^{16,17}$.
En niños, la principal causa de brotes de diarrea nosocomial es rotavirus, por lo tanto éste debería ser el primer agente a buscar en los servicios clínicos pediátricos.

Los resultados encontrados reafirman la recomendación de no solicitar CP en pacientes con más de tres días de hospitalización; e incluso, a la luz de nuestros datos se sugiere instaurar un punto de corte a los dos días de hospitalización, ya que no habría un impacto clínico significativo y permitiría ahorrar hasta $41 \%$ de estos exámenes, disminuyendo así los costos correspondientes para el paciente y para la administración pública, sin alterar el cuidado del paciente.

\section{Resumen}

Estudios han mostrado que es inapropiado realizar coprocultivos (CP) en pacientes con más de tres días de hospitalización, dado que la causa de diarrea no es atribuible a los patógenos entéricos que habitualmente se buscan en este examen ${ }^{1-3}$.

El objetivo de este trabajo es analizar el rendimiento de los CP realizados en pacientes hospitalizados en la Red de Salud UC desde enero a diciembre del 2002 y la proporción de resultados positivos obtenidos según los días de hospitalización, para así poder definir si las normas internacionales se adaptan a la realidad nacional.

Se recibieron $3474 \mathrm{CP}$, de los cuales $458(13,2 \%)$ provenían de pacientes hospitalizados. De éstos, 16 $(3,5 \%)$ fueron positivos, 13 se tomaron el día del ingreso, 2 al segundo día y 1 al quinto día de hospitalización. El rendimiento de $\mathrm{CP}$ realizados en pacientes con más de dos días de hospitalización fue sólo de $0,5 \%(1 / 190)$.

Los resultados encontrados reafirman la recomendación internacional de no solicitar $\mathrm{CP}$ en pacientes con más de tres días de hospitalización; e incluso, a la luz de los datos anteriores, se podría sugerir instaurar un punto de corte a los 2 días de hospitalización, ya que más allá de este tiempo no hay un impacto clínico significativo y podríamos ahorrar un $41 \%$ de estos exámenes, con los costos correspondientes.

\section{Bibliografía}

1.- Siegel D, Edelstein P, Nachamkin I. Inappropiate testing for diarrheal diseases in the hospital. JAMA. 1990; 263: 979-82.

2.- Morris A, Wilson M, Reller B. Application of rejection criteria for stool ovum and parasite examinations. J Clin Microbiol 1992; 30: 3213-6.

3.- Valenstein P, Pfaller M, Yungbluth M. The use and abuse of routine stool microbiology. A College of American Pathologist Q-Probes Study of 601 Institutions. Arch Pathol Lab Med 1996; 120: 206-11. 
4.- Román R E, Wilhelmi de Cal I, Cilleruelo M L, Calvo C, García M L, Sánchez-Fauquier A. Nosocomial gastroenteritis and asymptomatic rotavirus and astrovirus infection in hospitalized children. Ann Pediatr 2004; 60: 337-43.

5.- Comité de Microbiología Clínica. Sociedad Chilena de Infectología. Síndrome Diarreico Agudo: Recomendaciones para el diagnóstico microbiológico (consenso). Rev Chil Infect 2002; 19 (2): 101-13.

6.- Barbut F, Corthier G, Charpak Y, Cerf M, Monteil H, Fosse T, et al. Prevalence and pathogenicity of Clostridium difficile in hospitalized patients. A French multicenter study. Arch Intern Med 1996; 156: 1449-54.

7.- Rohner P, Pittet D, Pepey B, Nije-Kinge T, Auckenthaler R. Etiological agents of infectious diarrhea: implications for requests for microbial culture. J Clin Microbiol. 1997; 35: 1427-32.

8.- Navarro J, Rodenas G, Rodríguez J. Clinical evaluation of the stool culture in acute diarrhea. An Esp Pediatr 1989; 30 (6): 457-62.

9.- Soplan J, Fineberg H, Ferraro M J, Rosemberg M. Value of stool cultures. Lancet. 1980; August: 413-17.
10.- Chitkara Y, Mc Casland K, Kenefic L. Development and implementation of cost effective. Guidelines in the Laboratory Investigation of Diarrhea in a Community Hospital. Arch Intern Med 1996; 156: 1445-9.

11.- Selenite Broth - Selenite-F Broth. Zimbro MJ, Power DA (editors). Difco \&BBL Manual. Manual of Microbiological Culture Media, first ed. Maryland. Becton, Dickinson and Company. 2003. p. 508-9.

12.- Farmer J J III. Enterobacteriaceae: Introduction and Identification. Murray P, Baron EJ, Jorgensen J, Pfaller M, Yolken R (editors). Manual of Clinical Microbiology, $8^{\text {th }}$ ed. Washington DC. American Society of Microbiology (ASM Press) 2003; 636-53.

13.- Valenzuela M E, D’Ottone K. Diagnóstico presuntivo rápido de enteritis asociada a Campylobacter. Rev Chil Infect 1984; 2: 132-4.

14.- Bauer T M, Lalvani A, Fehrenbach J, Steffen I, Aponte J J, Segovia R, et al. Derivation and validation of guidelines for stool cultures for enteropathogenic bacteria other than Clostridium difficile in hospitalized adults. JAMA 2001; 285: 313-9.

Correspondencia a:

Patricia García Cañete

pgarcia@med.puc.cl 